\title{
A CD-ROM Retrieval System with Multiple Dialogue Agents
}

\author{
Keiichi Sakai, Tsuyoshi Yagisawa, and Minoru Fujita \\ Canon Inc., Media Technology Laboratory \\ 890-12 Kashimada, Saiwai-ku, Kawasali, 211, Japan \\ \{keiichi, yag428, minoru\}@cis. canon.co.jp
}

\begin{abstract}
In this paper, we proposed a new dialogue system with multiple dialogue agents. In our new system, three types of agents: a) domain agents, b) strategy agents, and c) context agents were realized. They give the following advantages to the user:

- the domain agents make the user aware of the boundary between the domains.

- the strategy agents make the uscr aware of the difference between the strategies.

- the context agents help the user to deal with multiple goals.

We expect that the complex behaviors of the system will become more visible to the user in different situations. The experimental results show that the user can retrieve effectively and obtain the expected goals easily by using these multiple agents.
\end{abstract}

\section{Introduction}

Recently, research into 'multi-agent system' is increasing. The multi-agent system is now one of the promising solutions to achieve a complicated system (Maes, 91; Nishida and Takada, 93; Nagao and Takeuchi, 94).

The multi-agent system which simulates cooperation between 'human-agents' is realized by an integration of simplified autonomous functions. And as a result it achieves a complicated system in total. It also has a latent potential to make a very flexible system.

Thus, we believe that if we introduce the concept of the multi-agent system into a dialogue system, we are able to construct a more soplisticated system which is able to treat various linguistic phenomena and to understand or to solve more complicated problems.

Focusing on dialogue systems, while most current dialogue systems can treat only one domain (a small world for a single service), some research(Goddeau et al., 94; Namba et al., 94) which aims at increasing the domains, what is called a transportable system(Grosz, 83; Paris, 89) are now on-going. In such systems, information retrieval across multiple domains is realized using the relational databases. However in our system, it is difficult to retrieve information across multiple domains, because the information is retrieved from CD-ROMs in which a large amount of texts are contained, by using full-text retrieval techniques.

And while there are robust and useful strategies in certain goals, there isn't an all-powerful sterategy which covers all goals. If a robust strategy in a certain goal is introduced into the system, the user misunderstands that the system has an allpowerful strategy. Thus, in our system the user sometimes gets into trouble as follows:

- the user misunderstands that the information contained across several data sources can be obtained at once.

- the user is confused between a certain retrieval strategy which is robust in a certain goal and another simple but rather redundant strategy.

Furthermore, it is difficult to manage a discourse involving multiple goals in current dialogue systems. This is because most current systems aren't robust enough for anaphora and they are able to manage only a single and simple context. This sometimes causes the following problem:

- the user has to manage the multiple contexts involving multiple goals, because the system only manages a single context. And, this makes it hard for the user to use the system.

As the result, the user also gets lost in the system.

In this paper, we focus on how to make the user aware of what the system can or cannot do. Thus, we propose a new dialogue system with multiple agents, in which we introduce the concept of multi-agent system into our dialogue system. In our system, three types of dialogne agents are realized: 1) for each domain, 2) for each strategy and 3) for each context. These agents take turns and play their roles according to the discourse sitnations. With these agents, our system will have the following characteristics:

- the domain agents make the user aware of the boundary between unintegrated domains.

- the strategy agents make the user aware of the difference between the domain oriented strategies. 
- the context agents make it easy for the user to deal with the complicated discourse involving multiple goals.

In this paper, we first explain our bascline spoken dialogne system TARSAN which deal with multiple domains. Secondly, we describe the problems which arise when we extend the system into multiple domains. After that, we propose a new dialogue system with multiple dialogue agents. We also describe the results of the examinations on the proposed system. Finally, we conclude the paper.

\section{The baseline system: TARSAN}

We have been constructing a spoken dialogue system which retrieves information from a large amount of texts contained in CD-ROMs, named TARSAN(Sakai et al., 94; Sakai et al., 95). Figure 1 shows the configuration of the baseline system TARSAN for multiple clomains.

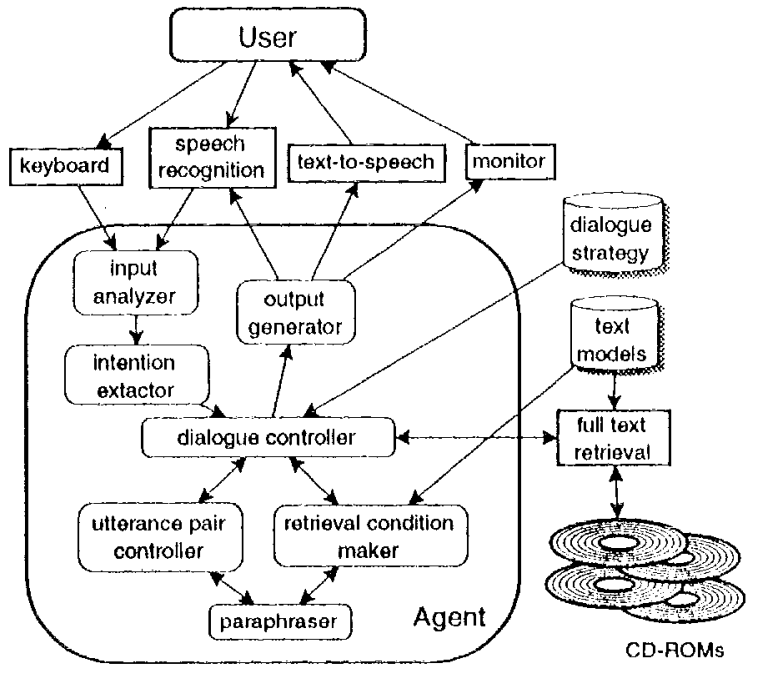

Figure 1: The configuration of 'TARSAN for multiple domains

TARSAN retrieves the information using the following processes:

1. The input analyzer analyzes the result of the spech recognition or the sentence received from keyboard.

2. 'The intention extractor extracts the ner's intention (i.e. question, answer, condition change, and so on) based on the analysis of the modality.

3. The utterance pair controller deals with not only a simple pair of $Q A$ but also deals with follow up questions based on utterance pair controllings.

4. The retrieval condition maker makes retrieval conditions which is sent to the full text retrieval process by the dialogue controller de. scribed below. 'The retrieval conditions are created by referring the 'text-models', which define the relation between the input words and the retrieval conditions.

5. The paraphraser translates various expressions of the inputs into a single domain oriented concept.

6. The dialogue controller determines the systen's behavior (to retrieve and to answer the result, or to request more retrieval conditions to the user) by referring the retrieval conditions and the dialogue strategy.

7. The output generator generates the output sentence to be announced by the text-tospeech process and the information to be displayed on the monitor.

Our current system TARSAN is able to access the following four CID-ROMs:

CD-ROM1: sight-secing information in Japan (i.c. name, location, explanation, and so on of tcmples, hot springs, golf courses, and so on)(Liosaido, 90).

CD-ROM2: hotel information in Japan (i.e. name, telephone number, room charges, equipment, and so on)(JTB, 92).

CD-ROM3: Japanese and foreign cinema information(i.e. title, cast, director, story, and so on) (PIA, 90).

CD-ROM4: Japanese professional baseball player information(i.e. name, belonging team, records, and so on)(Nichigai, 90).

IARSAN treats CD-ROM1 and 2 as a single travel domain, CD-ROM3 as a cinema domain, and CD-ROM4 as a baseball domain.

\section{Problems}

As we described in the introduction, we have ar dressed three main problems in our dialogue system. Two problems derive from the extension of the system to multiple donains. And the last one derives from the single path contextual management;.

1. The first problem is that the user misunder:stands that the information contained across several data sources can be obtained by a single input sentence. 'The following are examples of requests accross domains: The first example is contained in the cinema domain and in the travel domain, and the second exanple is contaned in the baseball domain and in the cinema domain.

Fxample 1: "Yamaguchi Momoc ga shren sita eiga no butai ni natta onsen wo shiritai."

(I want to know the hot spring which is the scenc of the cincma whose star is Yamaguchi Momoe.)

Wxample 2: "Puro yakyuu scnshu datia haiyuu ga shutsucn sita ciga wo oshiete."

(Tell ane the cincma where an actor who was a profossional baseball player performs.) 


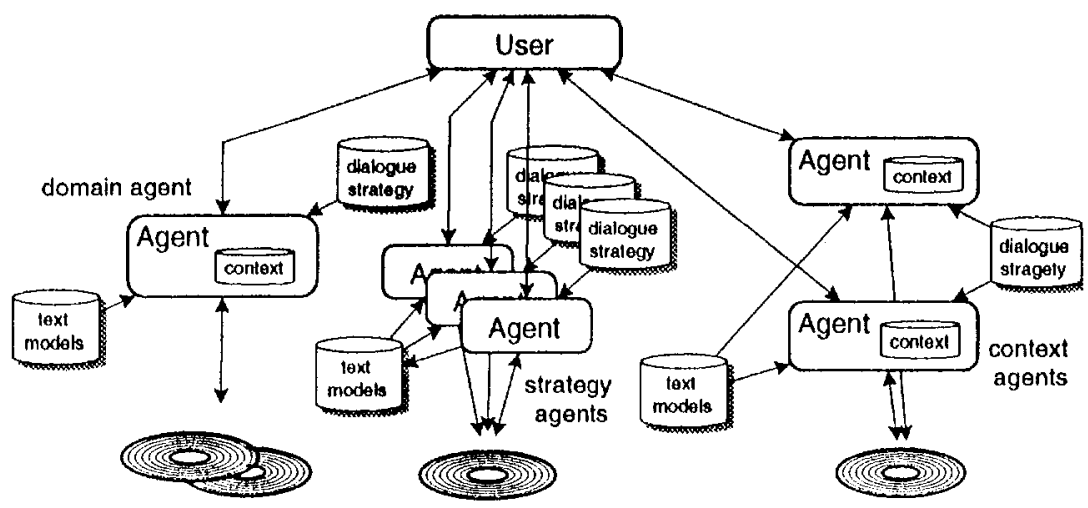

Figure 2: Three types of agents

2. The second problem is that the user misunderstands that the system has an all-powerful strategy, if it has a robust strategy for a certain purpose. Suppose that several discourse strategies exist in a single dialogue agent: one is a very sophisticated but very goal specific strategy which allows the user to reach the goal immediately, and another is a very simple but redundant strategy which has the ability to achicve any kind of goal. In this case, the user may confuse the potential of these strategies and feel uncomfortable about the gap.

3. The last problem is that the user has to manage multiple contexts concerning to multiple goals, because the system isn't cnough robust for anaphora and only manages a single context. And this makes it hard for the user to use the system. Table 1 is an example that the user compares the information between Hakone and Nikko'. The example shows that the user has managed the context himself, which seems very complicated.

We have also assumed that these three problems arise because the system only has a single dialogue agent. A single dialogne agent usually deals with everything and this makes the user invisible what the system can or cannot do. Thus, we propose a new dialogue system with multiple agents which make the system's ability more visible to the user.

\section{Dialogue system with multiple dialogue agents}

In this section, we introduce a new dialogue system with multiple dialogue agents. The purpose is to make the user aware of what the system can or cannot do. In our system, three types of dialogue agents are realized: 1) for each domain, 2) for each strategy and 3) for the each context. Here, we call these agents as 1) domain agents, 2) strategy agents, 3) context agents, respectively. Figure 2 shows a brief sketch of these three types of agents. These agents take turns and play their

\footnotetext{
${ }^{1}$ They are famous sight-seeing places in Japan.
}

Table 1: An example that the user manage the multiple-goals by oneself

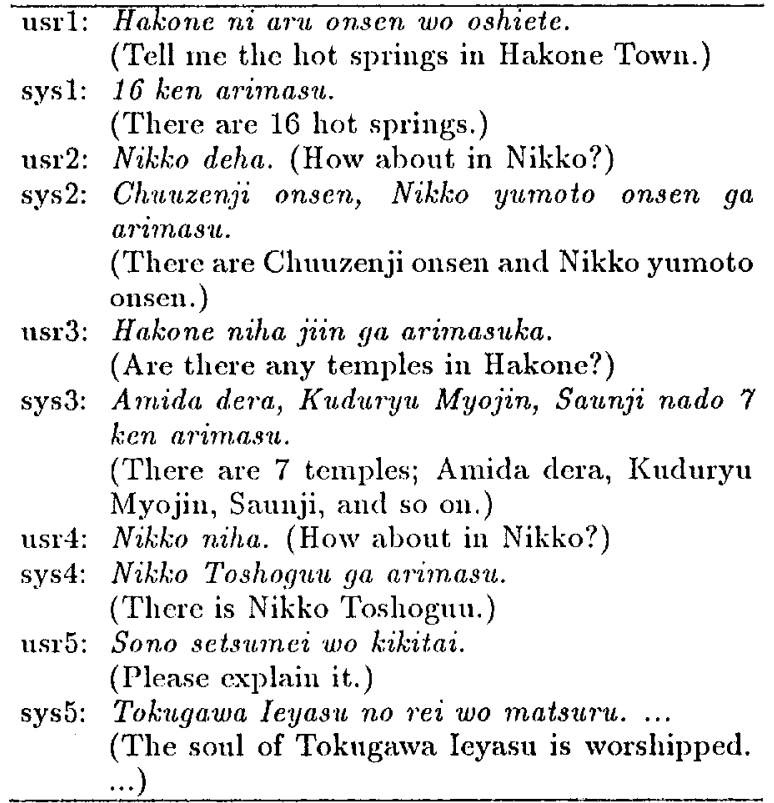

roles according to the discourse situations. The details of these agents are as follows.

\subsection{The domain agents}

To solve the first problem, we realized domain agents which perform information retrieval in each different domain. Figure 3 shows a brief sketch of the domain agents. The domain agents perform the basic interaction between the user and the system to retrieve the information in the basic manner specific to each domain. In every domain agent, indispensable and basic conditions for information retrieval are defined. Using these conditions, the domain agent communicates with the user and performs the information retrieval. And when the user's input moves from one domain to another domain, the domain agent will also change. Thus with the domain agents, the user is made aware of the boundary between the domains. We expect this mechanism to prevent the user from asking the question across uninte- 


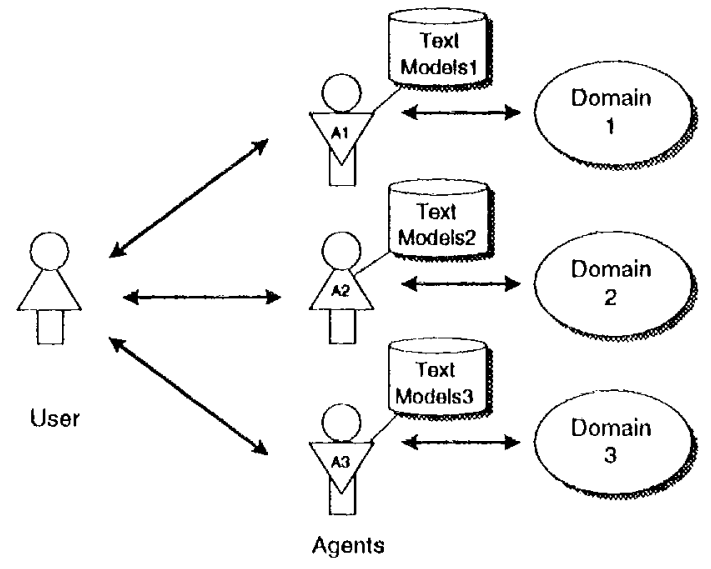

Figure 3: The domain agents

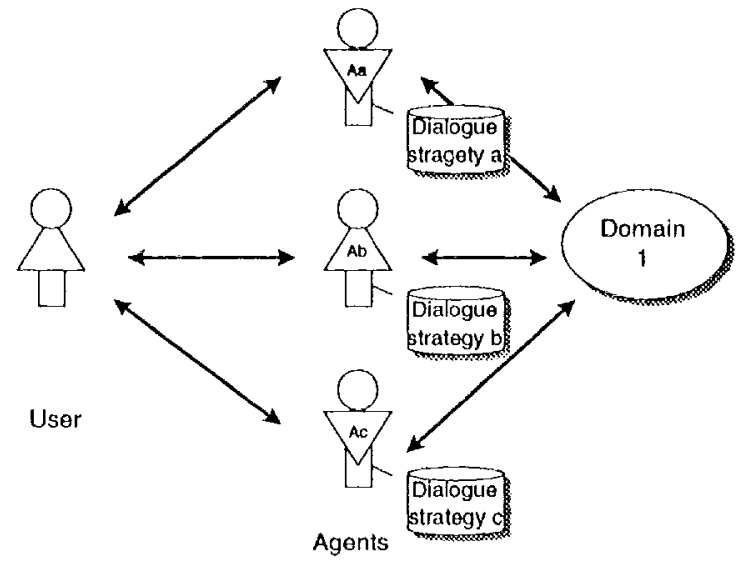

Figure 4: The strategy agents

business trip strategy agent: indispensable condition for the input is the destination, and the optional conditions are the room charge and the circumstances. When the optional conditions are not defined by the user, the strategy agent will recommend some choices to the user. The default responses are the name of the hotel and its telephone number in this task.

recreation strategy agent: indispensable condition for the input is the recreation equipment and the number of participants and the other conditions are optional. When the optional conditions are not defined by the user, the strategy agent will recommend some choices to the user. The default responses are also the name of the hotel and its telephone number in this task.

These strategy agents not only allow the user to use the system easily but also help the user to be aware of the characteristies of the dialogue strategy specific to the task.

Table 3 compares the difference between using the domain agent for travel and the business trip strategy agent. As you can see from the table, more fricndly discourse is achieved when using the strategy agent.

\subsection{The context agents}

To solve the last problem, we realized the context agents which perform the information retrieval dependent in different contexts. The context agent is defined when the user moves from one context to another. Figure 5 shows a bricf sketch of the context agents. Using the context agents the user can easily compare the results relating to multiple goals.

Table 4 is the dialogue which aims at the same goal as table 1 by using multiple context agents. As you can sec from the table 1 and 4 , more simplificel discourse is achieved when using context agents.

\footnotetext{
${ }^{2}$ Travel agent is able to retrive and find "the hot spring which is the scene of Izu no odoriko".
} 
Table 3: The comparison between the discourse using the domain agent and the strategy agent domain agent for trave

agt0: Hai, Ryokou eejent desu. (Hello, I'm travel agent.)

usr1: Tottori shi no hoteru wo shiritai. (I want to know the hotels in Tottori city.)

agt1: 34 ken arimasu. (34 rows exist.)

usr2: Ekimae ni aru hoteru. (Land circumstance is near the station.)

agt2: 8 ken arimasu. (8 rows exist.)

usr3: shukuhaku ryou ga 8000yen ika deha. (And room charge is under $8000 y e n$. )

agt3: Hoka no jouken "Ricchi jouken ga ekimae" wo nokoshi masuka.

(Do you kecp) another condtion, "Land circumstance is near the station"?)

usr4: Hai. (Yes.)

agt4: $A A A$ hoteru, hoteru $B B B$ desu. (AAA hotel and hotel BBB exist.)

usr5: Sono denwabangou wo oshiete. (Tell me the telephone numbers.)

agt5: $A A A$ hoteru no denurabangou ha $X X X$. $X X X X$, hoteru $B B B$ no denwabanou ha $Y Y Y-Y Y Y Y$ desu.

(The telcphone number of AAA hotel is $X X X-X X X X$, and the one of hotel BBB is YYY-YYYY.)

\begin{tabular}{l} 
business trip strategy agent \\
\hline agt0: Hai, Shucchou eejento desu. Dochira ni \\
shucchou nasai masu ka. \\
(Hello, I'n business trip agent. Where are \\
you goingo to go?) \\
usr1: Tottori shi desu. (Tottori city.) \\
agt1: Ricchi jouken ha ekimae, shukuhaku ryou ha \\
8000yen ika de yoroshii desuka. \\
(Circumstance is near the station, and room \\
charge is under 8000yen. Are these OK?) \\
usr2: Hai. (Yes.) \\
agt2: $2 k e n$ arimasu. AAA hoteru no denwabangou \\
haXXX-XXXX, hoteru BBB no denwabanou \\
ha YYY-YYYY desu. \\
(2 hotels exist. The telephone number of \\
AAA hotel is XXX-XXXX, and the one of \\
hotel BBB is YYY-YYYY.)
\end{tabular}

\section{Examinations}

In this section, we described the examinations of the proposed system. In order to examine the effectiveness of the multiple dialogue agent system (new system), we compare it with the single dialogue agent system (old system). Here the single dialogue agent is the domain agent for the travel domain.

We evaluated the system by counting the number of the interactions between the user and the system (Turns), the number of input characters of the users (Characters), and session time (Seconds) that subjects took to reach the same goal with new system and the old onc. Eight subjects examined these systems. They are all typists, but novices with dialogue systems. They were given a brief explanation of both systems and practiced on them for about quarter an hour each. We divide

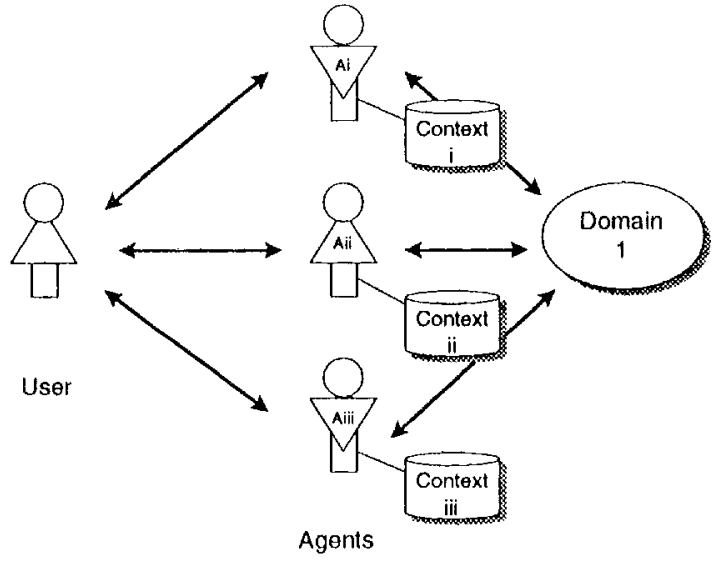

Figure 5: The context agents

Table 4: The dialogue using two context agents (to Hakone agent and Nikio agent)

11sr1: Onsen wo shiritai.

(I want to know the hot springs.)

H.agt1: 16 ken arimasu.

(There are 16 hot springs.)

N.agt1: Chuuzenji onsen, Nikko yumoto onsen ga arimasu.

(There are Chunzenji onsen and Nikko yumoto onsen.)

(to both agents)

1sr2: Jiin ha arimasuka

(Are there any temples?)

H.agt2: Amida dera, Kuduryu Myojin, Saunji nado 7 ken arimasu.

(There are 7 temples; Amida dera, Kuduryu Myojin, Saunji, and so on.)

N.agt2: Nikko Toshoguu ga arimasu. (There is Nikko Toshogut.)

(to Nikko agent)

usr3: Sono setsumci wo kikitai. (Please explain it.)

N.agt 3: Tokugaua Ieyasu no rei wo matsuru. ... (The soul of Tokugawa leyasu is worshipped. ...)

the subjects into two groups. Group 1 examined new system first and old one next, and group 2 did old system first and new one next.

\subsection{Examination 1}

The following goal is given to every subject:

Goal 1: You will go to Kurashiki City on business. Find a suitable hotel ${ }^{3}$. (Yon may select different hotels with each system.)

The relevant agent in the new system is the business trip agent. Table 5 shows the results (averages of Turns, Characters, and Seconds) of examination 1. These results show not only that both groups needed less dialogue using new system than using old system, but also that group 1 needed less dialogue, especially less session time (360:640), when they used old system than group 2. This

\footnotetext{
${ }^{3}$ There are 41 hotels in Kurashiki City.
} 
means that the user is able to learn how to use the old (strategy-less) system by using new system with a typical strategy. We also mention that all six subjects who selected different hotels were happy about the hotel using the new system.

Table 5: 'The results of examination 1

\begin{tabular}{c|cc|cc}
\hline & old & $\rightarrow$ new & new & $\rightarrow$ old \\
\hline Turns & 7.3 & 3.0 & 3.5 & 5.5 \\
Characters & 75 & 18 & 25 & 56 \\
Seconds & 640 & 175 & 190 & 360 \\
\hline
\end{tabular}

\subsection{Examination 2}

The following goal is given to every subject:

Goal 2: You have to select Kanazawa or Sendai for sight-sceing. Compare them using some retrieved information, and select one.

The relevant agents in the new system are Kanazawa agent and Sendai agent. Table 6 is the results of examination 2. These results show an interesting phenomenon that in the case of the dialogue comparing multiple goals with these complicated processes, the user tends to stop comparing by session time (from five minutes to ten minites) in favour of the obtained retrieval results. And the new system is able to obtain more retrieval results than the old system. Thus the new system is better than the old system in the case of dealing with multiple goals.

Table 6: The results of examination 2

\begin{tabular}{c|cc|cc}
\hline & \multicolumn{2}{|c|}{ old $\rightarrow$ new } & \multicolumn{2}{|c}{ new $\rightarrow$ old } \\
\hline Turns & 7.0 & 10.3 & 9.3 & 8.5 \\
Charactcrs & 79 & 54 & 51 & 96 \\
Seconds & 442 & 420 & 458 & 526 \\
\hline
\end{tabular}

\section{Conclusion}

In this paper, we proposed a new dialogue system with multiple clialogue agents. In our new system, three types of agents were realized. 'They were a) domain agents, b) strategy agents, and c) context agents. These agents give the following advantages to the user:

- the domain agents prevent the user from asking the questions across unintegrated domains.

- the strategy agents make the nser aware of the difference between the domain oriented strategies.

- the context agents make it easy for the user to deal with the complicated discourse involving multiple goals.

Using these agents, we expect the user to understand what the system can or cannot do. The experimental results show that the user can retrieve effectively and obtain the expected goals easily by using these multiple agents.

\section{Acknowledgement}

The anthors wish to thank Dr. Hideyuki Tamura, head of the Media Technology Lab., for giving the opportunity of this study, Dr. Yasuhiro Komori and Tom Wachtel for suitable advice in translating this paper into English, and members of the Intelligent Media Div. for uscful discussions.

\section{References}

Cécile L.Paris. 1989. The Use of Explicit User Models in a Generation System for Tailoring Answers to the User's Level of Expertise. User Models in J)ialog Systems, pp.200 pp.232. Springer-Verlag, A.Kobsa, W.Wahler (Eids.).

Goddeau D)., Brill F., Glass J., Pao C., Phillips M, Polifromi .J., Seneff S., and Zue V. 1994. Galaxy: A Human-language Interface to Online Travel Information. In Procedings of ICSLP-94, S13-11.

Grosz B.J. 1983. A Transportable NaturalIanguage Intcrface System. In Procecdings of Conference on Applied Natural Language Processing, pp.25- pp.30.

JTB. 1992. JTB's Accommodation Information (Flectronic Book YRRS-094). el. by Nakajime R. JTB. Tokyo.

Kosaiclo. 1990. TABIGURA (Electronic Book YRRS-028). ed. by Nilon-Kanko-Kyokai Corporation. Tokyo.

Maes P. 1991. The Agent Network Architecture (ANA). SIGAR'I bulletin (2), 4.

Nagao K. and Takenchi A. 1994. Social Interaction: Multimodal Conversation with Social Agents. In Proceedings of AAAI-94, pp. 22-28.

Namba Y., Tsuji H., and Kinugawa H. 1994. Natural Language Interface for Multiple Systems Secucutial Control (In Japanese). IPSJ Vol.35 No.1, p. 20 34.

Nichigai. 1990. The professional baseball (Electronic Book YRRS-(023). Morioka H. and Nichigai Asscociates Co. Tokyo.

Nishicla T. and Takeda H. 1993. Towards the Knowledgeable Community. In Proceedings of International Conference on Building and Sharing of Very-Large: Scale Knowledge Bases '93 (KB \& $K S$ '93), pp. 157-166. Ohmsha.

PIA. 1990. PIA cinema club (Electronic Book YRRS-016). ed. by Watanabe K. PIA Co.

Sakai K., Ikeda Y., and Fujita M. 1994. Robust Discourse Processing Considering Misrecognition in Spoken Dialogne System. In Proceedings of ICSLP-94, S17-7.

Sakai K., Ikeda Y., and Fujita M. 1995. Gaidansu Sisuternu no Maruchi Domein-ka no Kokoromi (In Japanese), (A trial to Increase Domains in Guidance System). In Proceedings of The First Annual Meeting of The Association for Natural Language Processing, pp. 301 304. 